\title{
COMPUTATION OF DISPLACEMENTS FOR NONLINEAR ELASTIC BEAM MODELS USING MONOTONE ITERATIONS
}

\author{
PHILIP KORMAN \\ Department of Mathematical Sciences \\ University of Cincinnati \\ Cincinnati, Ohio 45221 \\ (Received September 2, 1986)
}

ABSTRACT. We study displacement of a uniform elastic beam subject to various physically important boundary conditions. Using monotone methods, we discuss stability and instability of solutions. We present computations, which suggest efficiency of monotone methods for fourth order boundary value problems.

KEY WORDS AND PHRASES. Monotone schemes, elastic beam, existence and approximation, stability.

1980 AMS SUBJECT CLASSIFICATION CODES. 34,65 .

\section{INTRODUCTION}

We study the displacement curve $u=u(x)$ of a uniform elastic beam of length $\ell$, supporting a distributed load of intensity $q(x, u(x))$. This load causes the beam to bend from its equilibrium configuration along the $x$-axis. For small displacements we have:

$$
u^{\prime \prime \prime}=\frac{q(x, u)}{E I} \equiv f(x, u) \quad, \quad 0<x<\ell,
$$

where $E$ is Young's modulus, I is the moment of inertia, see e.g. [1]. That is we study the equation (1.1) with appropriate two-point boundary conditions. We show that the monotone iteration scheme and other monotone methods are applicable and provide an effective computational tool, as well as means of proving existence theorems.

Monotone methods are usually associated with maximum principles. Clearly, there is no weak maximum principle for $u^{\prime \prime \prime}=f(x)$, since condition $f(x)>0$ does not preclude $u(x)$ from having extreme points inside of any interval. However, if we add the boundary conditions

$$
u(0)=\alpha, \quad u^{\prime}(0)=\beta, u(\ell)=\gamma,-u^{\prime}(\ell)=\delta
$$

with $\alpha, \beta, \gamma, \delta>0$, then condition $f(x)>0$ does imply $u(x)>0$ (since the Green's function in (2.2) is positive). This is an example of inversepositivity, a property of boundary-value problems, rather than of equations, see $[2,3]$. In [3] we applied monotone methods to general inverse-positive problems, 
including (1.1) - (1.2). In this paper, we present some further results, and report on computations with numerous nonlinearities $f(x, u)$. The main results of this note are the theorem 3 , and our discussion of stability leading to the theorem 4. Theorems 1 and 2 are essentially known, and are illustrated here computationally. For a previous application of monotone methods for this model, see J. Schroder [4], where a rather involved splitting method was used. Most of the results in this paper were stimulated by computations (and the fast convergence that we encountered).

Our results apply to other physically important boundary conditions, see Remark 2, as well as to biharmonic equations in higher dimensions, see [3].

Throughout the paper $\psi$ will denote subsolution, $\phi$-supersolution, $|u|_{C^{\circ}}=\max _{0<x<\ell}|u(x)|$.

2. GENERAL RESULTS

The following theorem was proved in [3].

Theorem 1. Consider the problem (one-dimensional)

$$
\begin{gathered}
u^{\prime \prime \prime}=f(x, u), 0<x<\ell \\
u(0)=\alpha, u^{\prime}(0)=\beta, \quad u(l)=\gamma,-u^{\prime}(l)=\delta .
\end{gathered}
$$

Assume the following for $0<x<\ell$.

(i) There exists a supersolution $\phi \varepsilon c^{4}$, i.e., $\phi^{\prime \prime \prime}>f(x, \phi), \phi(0)>\alpha$, $\phi^{\prime}(0)>\beta, \phi(l)>\gamma,-\phi^{\prime}(l)>\delta$, and a subsolution $\psi(x)$ defined by reversing the above inequalities. Moreover $\psi<\phi$.

(ii) $f$ is continuous, increasing in $u$ for $\psi<u<\phi$.

Then the problem (2.1) has a $c^{4}$ solution $u(x)$, and $\psi<u<\phi$. Moreover, starting with $\psi$ or $\phi$ we get two monotone sequences of Picard iterations.

Theorem 2. For the problem (2.1) starting with some continuous function $u_{0}(x)$ define a sequence of approximations $\left\{u_{n}(x)\right\}$ by the formula

$$
\begin{aligned}
u_{n}(x) & =\alpha\left(1-\frac{3}{l^{2}} x^{2}+\frac{2}{l^{3}} x^{3}\right)+\beta x\left(1-2 \frac{x}{\ell}+\frac{x^{2}}{l^{2}}\right) \\
& +\gamma \frac{x^{2}}{l^{3}}(3 \ell-2 x)+\delta \frac{x^{2}}{l^{2}}(\ell-x) \\
& +\int_{0}^{\ell} G(x, \xi) f\left(\xi, u_{n-1}(\xi)\right) d \xi,
\end{aligned}
$$

where $G(x, \xi)$ is the Green's function for $u^{\prime \prime}$ " with the boundary conditions $u(0)=u^{\prime}(0)=u(l)=u^{\prime}(l)=0$, which is given by the formula (see [5])

$$
G(x, \xi)=\frac{1}{6 l^{3}} x^{2}(\xi-l)^{2}(3 \ell \xi-2 \xi x-\ell x) \text { for } x<\xi, G(x, \xi)=G(\xi, x) \text {. }
$$

Assume the following for $0<x<\ell$.

$$
u_{0}<u_{1}, u_{0}<u_{2} \text {. }
$$


(ii) $f(x, u)$ is continuous, decreasing in $u$ for $u_{0}<u<u_{1}$, and there is a constant $f_{0}>0$, such that $|f(x, u)-f(x, v)|<f_{0}|u-v|$ if $u_{0}<u, v<u_{1}$.

(iii) $f_{0} \frac{\ell^{4}}{384}<1$.

Then the problem (2.1) has a solution $u(x) \in c^{4},\left|u-u_{n}\right|_{c} \rightarrow 0$ as $n \rightarrow \infty$ (uniform convergence), and moreover

$$
u_{0} \leqslant u_{2} \leqslant u_{4}<\ldots<u<\ldots<u_{3} \leqslant u_{1} \text {. }
$$

Solution is unique in the order interval $\left[u_{0}, u_{1}\right]$.

Proof. Relations (2.4) easily follow by induction. To prove convergence write

$$
u_{n+1}(x)-u_{n}(x)=\int_{0}^{l} G(x, \xi)\left[f\left(\xi, u_{n}(\xi)\right)-f\left(\xi, u_{n-1}(\xi)\right)\right] d \xi,
$$

and hence

$$
\max _{0<x<\ell}\left|u_{n+1}(x)-u_{n}(x)\right|<f_{0} \max _{x} \int_{0}^{\ell} G(x, \xi) d \xi \max _{0<x<\ell}\left|u_{n}(x)-u_{n-1}(x)\right|
$$

An elementary computation shows that

$$
\max _{x} \int_{0}^{\ell} G(x, \xi) d \xi=\int_{0}^{\ell} G(\ell / 2, \xi) d \xi=\frac{\ell^{4}}{384} .
$$

To prove uniqueness it suffices to write the equation for the difference of two solutions $w(x)$, multiply it by $w$ and integrate $\int_{0}^{\ell}$ by parts twice.

Assume now that $f(x, 0) \equiv 0$ and $\alpha=\beta=\gamma=\delta=0$. Then (2.1) possesses a trivial solution $u \equiv 0$. Next, we discuss existence of a nontrivial solution.

Theorem 3. Lef $f(x, u)$ be continuous function increasing in $u$ for $0<u<\infty$, $0<x<\ell, f(x, u)>0$ for $u>0$, and (uniformly in $x$ ).

$$
\lim _{u \rightarrow 0^{+}} \frac{u}{f(x, u)}=\lim _{u \rightarrow+\infty} \frac{f(x, u)}{u}=0 \text {. }
$$

Then the problem (2.1) has a positive solution, provided $\alpha, \beta, \gamma, \delta>0$.

Proof. Let $\psi_{0}(x)>0$ and $\lambda_{0}>0$ be the principle eigenfunction and eigenvalue for $u^{\prime \prime \prime}=\lambda u, u(0)=u^{\prime}(0)=u(\ell)=u^{\prime}(\ell)=0$, whose existence is guaranteed by the Krein-Rutman theorem (see [3] for details). Let $\phi_{0}(x)$ and $\mu_{0}$ be the same for $u^{\prime \prime \prime}=\mu u, u(-\rho)=u^{\prime}(-\rho)=u(\ell+\rho)=u^{\prime}(\ell+\rho)$ with $\rho>0$. Notice, $\phi_{0}(x)>0$ for $0<x<\ell$. Now it is easy to check that $\psi=\varepsilon \psi_{0}(x)$ and $\phi=M_{0}(x)$ are sub and supersolutions, provided $\varepsilon, \rho$ are sufficiently small and $M$ is sufficiently large.

This theorem covers in particular the sublinear nonlinearities. For the superlinear case, we state the following conjecture.

Conjecture. Assume the conditions of the theorem 3 with (2.6) changed to 


$$
\lim _{u \rightarrow 0^{+}} \frac{f(x, u)}{u}=\lim _{u \rightarrow+\infty} \frac{u}{f(x, u)}=0 .
$$

Then the problem $(2.1)$ has a positive solution.

We expect this solution to be unstable. We discuss the concept of stability next.

For the equations of second order, like $\Delta u=f(x, u)$, it is known that if solution can be computed by the monotone iteration method, then it must be stable. The proof of this result, as well as the definition of stability itself, makes use of the maximum principle for parabolic equations, see e.g. [6]. Since we do not know of any maximum principle for $u_{t}+u_{x x x x}$, we have to generalize the concept of stability. We state it for a general inverse-positive operator $L$, see e.g. $[2,3]$; in our case $L u=u^{\prime \prime}$ " with the boundary conditions of $(2,1)$.

Definition. Solution $u(x)$ of $L u=f(x, u)$ with $f$ continuous and increasing in $u, x \varepsilon R^{n}$, is called stable if for any $\varepsilon>0$ there exist sub and supersolutions $\psi(x)$ and $\phi(x)$ with $0<|u-\phi|_{C^{\circ}}+|u-\psi|_{C^{\circ}}<\varepsilon$, such that $\psi<u<\phi$. (If this condition holds only with $\phi=u(\psi=u)$ we say that solution is stable from below (above)). Solution is called unstable if in the conditions above $\phi<u<\psi$.

It is easy to see that the concepts of stability and instability are mutually exclusive for an isolated solution. (Our concept of stability corresponds to the 'strong stability' in H. Matano [7], and it implies stability for elliptic equations of second order, see Proposition 4.2 in [7].)

Theorem 4. The positive solution of

$$
u^{\prime \prime \prime}=u^{p}, u(0)=u^{\prime}(0)=u(l)=u^{\prime}(l)=0, p>1
$$

must be unstable if it exists, and hence not computable by monotone iterations.

Proof. If $u$ is a solution, then $v=\varepsilon u$ satisfies $v^{\prime \prime}-v^{p}=\left(\varepsilon-\varepsilon^{p}\right) u^{p}$. Hence $v$ is a supersolution for $\varepsilon<1$, and a subsolution for $\varepsilon>1$.

On the other hand, it is easy to see that the trivial solution of (2.8) is stable from above (take $\psi=0, \phi=\varepsilon \psi_{0}$; if $p$ is an odd integer take $\psi=-\varepsilon \psi_{0}$, giving two-sided stability). Not surprisingly, it persists under small onesided perturbations.

Proposition 1. Consider the problem

$$
u^{\prime \prime \prime}=u^{p}+\varepsilon f(x, u), u(0)=\alpha, u^{\prime}(0)=\beta, u(l)=\gamma, u^{\prime}(l)=\delta .
$$

Here $f$ is continuous, increasing in $u ; f(x, u), \alpha, \beta, \gamma, \delta>0 ; f(x, 0) \neq 0$.

Then for $\varepsilon, \alpha, \beta, \gamma, \delta$ sufficiently small, the problem (2.9) has a nonnegative solution.

Proof. With $\psi_{0}$ as defined in the theorem 3, choose $\tau>0$ so small that $\tau \mu_{0} \psi_{0}>{ }_{\tau} P_{\psi_{0}}{ }^{\mathrm{P}}$ for $0<x<\ell$ (with $\rho>0$ chosen so small that $\psi_{0}(0)>0, \psi_{0}(l)<0$ ). After fixing $\tau$ we see that $\phi=\tau \psi_{0}$ is a supersolution for our problem, provided $\varepsilon, \alpha, \beta, \gamma, \delta$ are sufficiently small. Take $\psi=0$ for a subsolution, and apply the theorem 1 . 
In [3] we discussed some general non-existence results which can be applied to (2.1). In particular, we had the following result which will be illustrated computationally.

Proposition 2. Consider the problem $(p>1)$

$$
u^{\prime \prime \prime}=u^{p}+\lambda, u(0)=u^{\prime}(0)=u(l)=u^{\prime}(l)=0 \text {. }
$$

Then for $\lambda>\left(\frac{\lambda_{0}}{p}\right)^{\frac{p}{p-1}}(p-1)$ the problem (2.10) has no positive solution; $\lambda_{0}$ as defined in the theorem 3 is given by $\lambda_{0}=k^{4}$, $\ell k=4.7300$, see [5, p. 146$]$.

our next result provides a simple error estimate.

Proposition 3. For the problem (2.1) assume conditions of either theorem 1 or 2. Denote $G_{0}=\max _{0<x<\ell} \int_{0}^{\ell} G(x, \xi) d \xi=\frac{\ell^{4}}{384}$. Let $f(x, u)$ be Lipshitz in uniformly in $0<x<\ell$, with Lipshitz constant $f_{0}$ for $\psi<u<\phi\left(u_{0}<u<u_{1}\right)$ in case of theorem $1(2)$. In case of theorem 1 assume additionally that $G_{0} f_{0}<1$. If $u$ denotes the solution obtained by monotone iterations $\left(u_{0}=\psi\right.$ or $\phi$ in the theorem 1), then

$$
\left|u-u_{n}\right|_{C}<\frac{G_{0}^{n_{f}}{ }_{0}^{n}}{1-G_{0}^{n_{f}^{n}}}\left|u_{1}-u_{0}\right|_{C} \cdot
$$

Proof. Follows by writing $u-u_{n}=\sum_{k=n}^{\infty}\left(u_{k+1}-u_{k}\right)$, and estimating each term as in the theorem 2. (Notice that for the theorem 1 we get convergence of both monotone sequences to the same solution.)

Remark 1. In the conditions of the theorem 1 assume that $f=f(u), f(0)>0$. Then we can start with a subsolution $u_{0}=0$, compute $u_{1}=\frac{f(0)}{24} x^{2}(x-\ell)^{2}$, and hence by $(2.11)$

$$
\left|u-u_{n}\right|_{C^{0}}<\frac{f(0)}{24} \cdot \frac{e^{4}}{16} \frac{f_{0}^{n} G_{0}^{n}}{1-f_{0}^{n} G_{0}^{n}}=\frac{f(0) f_{0}^{n}}{1-f_{0}^{n} G_{0}^{n}}\left(\frac{e^{4}}{384}\right)^{n+1}
$$

which explains the fast convergence that we encountered in our experiments $(\ell=2)$. On the other hand, we see from (2.12) that convergence is slower, in general, for larger $\ell$, and it cannot be guaranteed beyond a certain value of $\ell$. This is no surprise, since e.g. the problem $u^{\prime \prime \prime}=u^{2}+1, u(0)=u^{\prime}(0)=u(\ell)=$ $u^{\prime}(\ell)=0$ has no solution for $\ell$ sufficiently large, as can be seen by rescaling $x=\ell \xi$, and then applying the theorem 4 in [3].

Remark 2. Finally, we mention that all our results hold for other physically important boundary conditions for $L u=u^{\prime \prime}$ ", since they only depended on the positivity of the Green's function. Next we list two such problems with corresponding Green's functions (see [5]), whose positivity is easy to check.

(I) $L u=u^{\prime \prime \prime}, u(0)=u^{\prime \prime}(0)=u(\ell)=u^{\prime \prime}(\ell)=0$. 


$$
\begin{aligned}
& G(x, \xi)=\frac{1}{6 \ell} x(\xi-\ell)\left(x^{2}+\xi^{2}-2 \ell \xi\right) \text { for } x<\xi, G(x, \xi)=G(\xi, x) . \\
& \text { (II) } L u=u^{\prime \prime \prime}, u(0)=u^{\prime}(0)=u(\ell)=u^{\prime \prime}(\ell)=0 . \\
& G(x, \xi)=\frac{x^{2}(\xi-\ell)}{12 \ell^{3}}\left[3 \ell \xi^{2}-6 \ell^{2} \xi+\left(-\xi^{2}+2 \ell \xi+2 \ell^{2}\right) x\right] \text { for } x \leqslant \xi, G(x, \xi)=G(\xi, x) .
\end{aligned}
$$

3. Numerical Experiments

To compute the solution of (2.1) we were using the formuld (2.2) with $u_{0}$ being either sub or supersolution, or as defined in the theorem 2 . In our examples we took $\ell=2, \alpha=\beta=\gamma=\delta=0$, and computed solution at 200 mesh points with the uniform step size $h=0.01$. The integral in (2.2) was approximated by the trapezoid rule. A PASCAL program was written, and the computations were performed with six (sometimes twelve) decimal digits; changing the function $f(x, u)$ required changing of only one line of code.

One remarkable thing common to all the experiments was fast convergence. For example, for $f=u^{2}+1$ and a subsolution $\psi=0$ it took just 5 iterations for all twelve decimal digits to stabilize (and the errors of integration in this and all other examples never interfered with monotonicity of iterations). The CPU time on VAX-11 was 6.2 seconds. To check that convergence is to actual solution, we started with a supersolution $\phi=0.05 x^{2}(2-x)^{2}$, and obtained the same answer in 5 iterations. Similar computations (with six decimal digits) were performed for other $f(x, u)$, and some of the results are presented in the Table 1. (The remainder term of the trapezoid rule can be easily estimated in each case. Indeed, estimating $u_{n+1}^{\prime \prime} \leqslant \int_{0}^{\ell} G_{x x}(x, \xi) f(\xi, \phi(\xi)) d \xi$, we can easily estimate $\frac{\partial^{2}}{\partial \xi^{2}}\left[G(x, \xi) f\left(\xi, u_{n}(\xi)\right)\right]$. In practice, however, it is much easier just to check that the iterations starting with $\psi$ and $\phi$ lead to the same result, and decrease $h$ if necessary).

When we tried $f=u^{2}+300$ with subsolution $\psi=0$, the iterations diverged $($ to $+\infty)$, which illustrates nonexistence of solution for this equation, in accordance with Proposition 2. We also considered $f=u^{2}+1-a u$. For constants $a=1$ and $a=10$ one gets alternating convergence as in (2.4) (starting with $u_{0}=0$ ), but for $a=100$ the iterations diverged. This is because the condition $u_{0}<u_{2}$ of the theorem 2 is violated.

What happens when $f(0)=0$, but one expects existence of a nontrivial solution (in addition to the trivial one). For $f(u)=10 \sqrt{u}$ we were able to compute a nontrivial solution, see Table 1 , thus illustrating the theorem 3 . For $f(u)=u^{2}$ our attempts to compute a nontrivial solution failed. This led us to Theorem 4, which shows that the positive solution must be unstable, if it exists, and hence not computable by monotone iterations.

Finally, we mention that by changing the Green's function, we performed similar computations for the problem $-u^{\prime \prime}=f(x, u), u(0)=u(l)=0$. We obtained similar results with considerably slower convergence. The reason is 
that our Green's function in (2.3) is "small". One measure of its smallness is given in (2.5). Another one is derived by an elementary computation:

$$
\max _{x, \xi} G\left(x, \xi_{,}\right)=G(\ell / 2, \ell / 2)=\frac{\ell^{3}}{192}
$$

Table 1. Accuracy $=10^{-6}$; $v$-subsolution, $\phi$-supersolution; number of $i$ terations refers to the larger one in case of two monotone sequences.

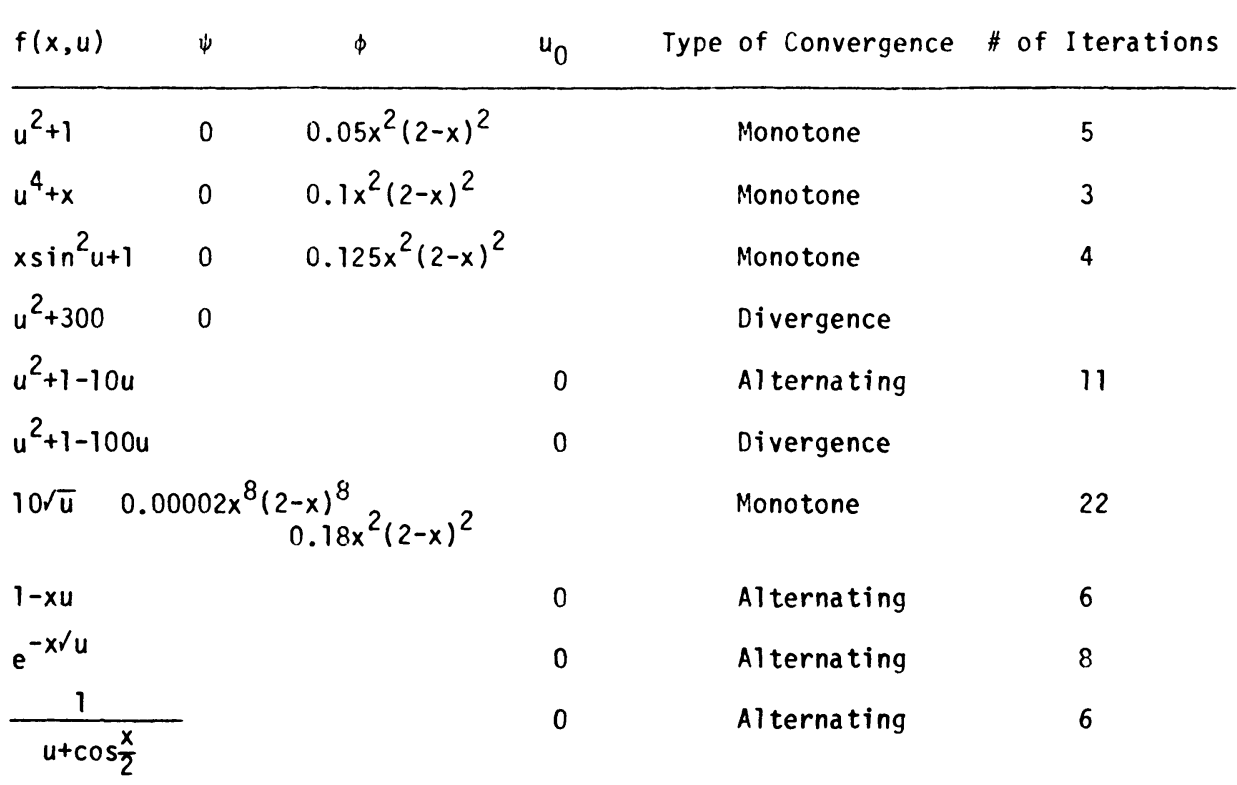

Acknowlegements. It is a pleasure to thank A. Leung and K. Meyer for useful
discussions.

\section{REFERENCES}

1. TIMOSHENKO, S. Strength of Materials, Van Nostrand (1955).

2. SCHRODER, J. Operator inequalities, Academic Press, New York (1980).

3. KORMAN, P. Existence and approximation of solutions of inverse-positive problems, to appear in Nonlinear Analysis.

4. SCHRODER, J. Fourth order two-point boundary value problems; estimates by two-sided bounds, Nonlinear Analysis 8 (1984), 107-114.

5. COLLATZ, Li Eigenwertprobleme und ihre numerische behandlung, Chel sea, New
York (1948).

6. SMOLLER, J. Shock waves and reaction-diffusion equations, Springer, New York
(1983). 
7. MATANO, H. Existence of nontrivial unstable set for equilibriums of strongly order-preserving systems, J. Fac. Sci. Univ. Tokyo 30 (1984), 645-673.

8. KORMAN, P. On application of the monotone iteration scheme to noncoercive elliptic and hyperbolic problems, Nonlinear Analysis 8 (1984), 97-105.

9. KORMAN, P. On application of monotone iteration scheme to wave and biharmonic equations, Proc. Sympos. Pure Math., vol. 45 (1986).

10. LAKSHMIKANTHAM, $V$. The present state of the method of upper and lower solutions, in Trends in Theory and Practice of Nonlinear Differential Equations, edited by V. Lakshmikantham, Marcel Dekker, New York (1984). 


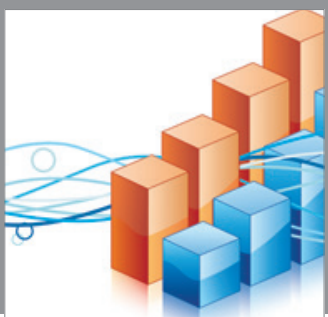

Advances in

Operations Research

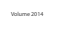

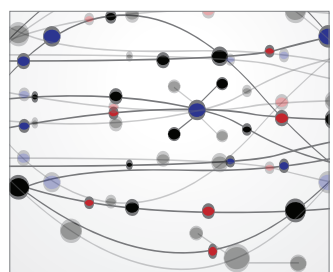

\section{The Scientific} World Journal
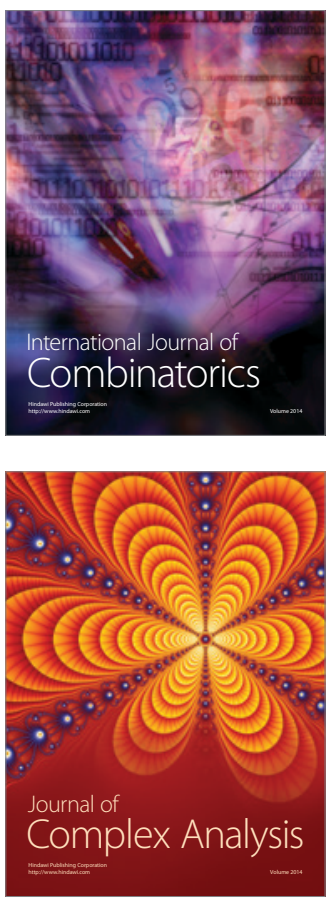

International Journal of

Mathematics and

Mathematical

Sciences
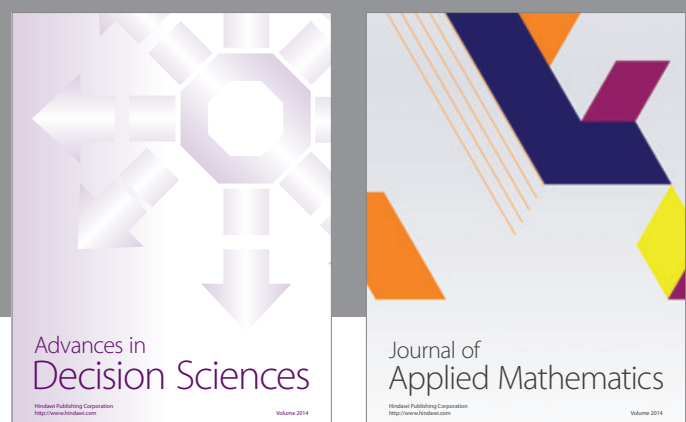

Journal of

Applied Mathematics
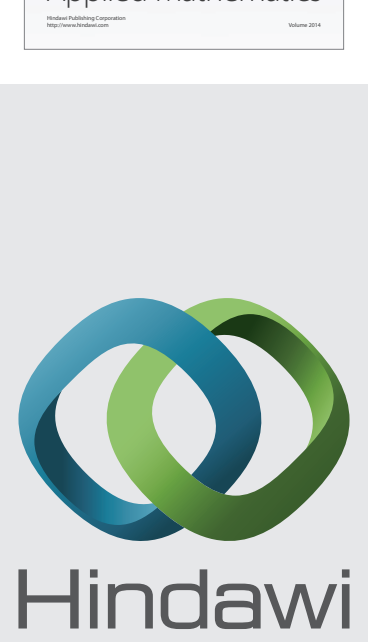

Submit your manuscripts at http://www.hindawi.com
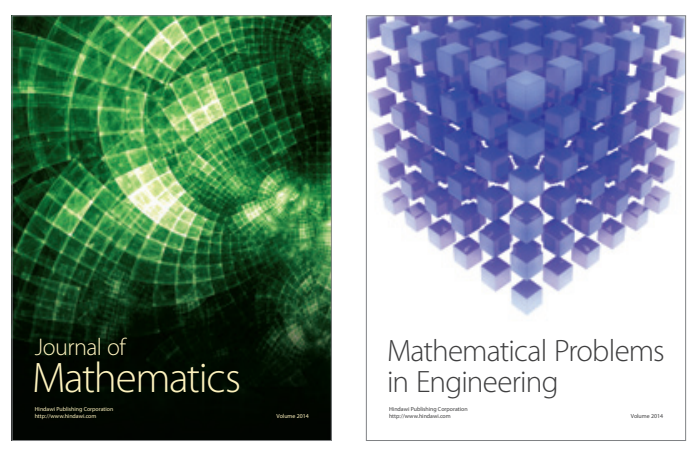

Mathematical Problems in Engineering
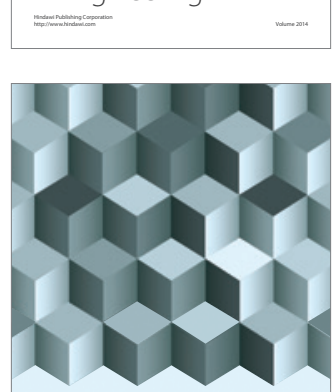

Journal of

Function Spaces
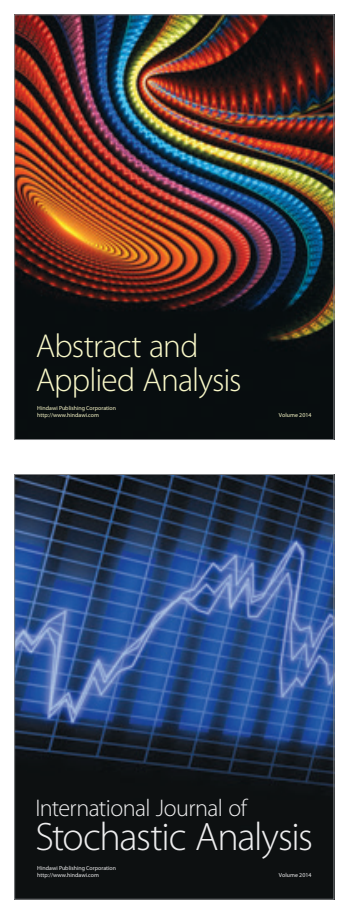

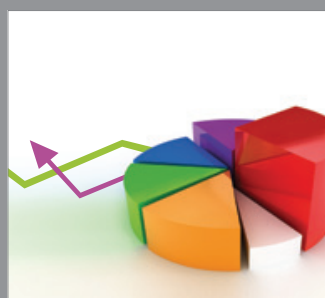

ournal of

Probability and Statistics

Promensencen
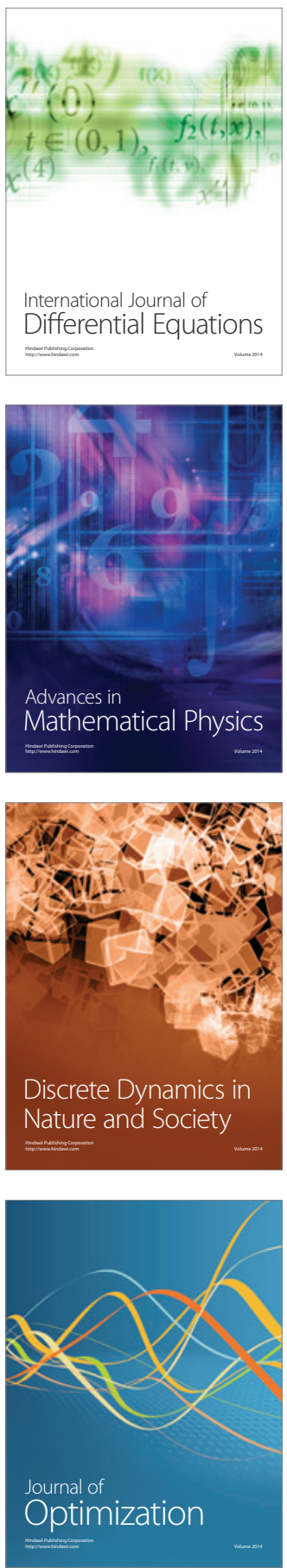